\section{$(\hat{B})>$ HORIZONTES}

www.revistahorizontes.org

\title{
El respeto a los animales como tema transversal en valores para educación inicial
}

\author{
Respect to animals as a transversal topic on values in initial education
}

O respeito ao animal como tema transversal nos valores da educação inicial

\section{ARTÍCULO GENERAL}

\author{
Ángel Carmelo Prince Torres \\ arbqto@gmail.com \\ ORCID: 0000-0002-0059-7797
}

Instituto Universitario Pedagógico “Monseñor Rafael Arias Blanco”, Venezuela

Recibido 06 de noviembre 2020 | Arbitrado y aceptado 22 de febrero 2021| Publicado en marzo 2021

\section{RESUMEN}

Los infantes durante los primeros años de sus vidas están en el momento ideal para cimentar las bases de su sistema de creencias, en consonancia con los objetivos sociales. Este trabajo tuvo como propósito desarrollar la importancia de la noción sobre respeto hacia los animales como tema transversal desde la educación inicial, de manera que se potencie en los niños un sistema de valores fundado en la empatía. El esquema investigativo utilizado fue documental con enfoque cualitativo y método hermenéutico. Con la información recabada se determinó que episodios de maltrato animal ocurren alrededor del mundo, y la educación es un instrumento que puede reconducir ese fenómeno porque los niños en el proceso de aprendizaje están prestos a construir conocimientos. Se concluyó que es importante que el niño dentro de educación inicial tenga nociones sobre las vidas de los animales, y esto ayudará a que desarrolle sensibilidad hacia la naturaleza.

Palabras clave: Respeto; animales; transversalidad; educación inicial; valores

\begin{abstract}
Children can consolidate, in their first years of existence, their beliefs according to the goals of the society. This paper developed the importance about the notion of respect to the animals as a transversal topic from initial education. This investigation is documental with a qualitative approach and a hermeneutic method. The information that was gathered, determined that episodes of animal cruelty happen all around the world and because of that, education is a tool which can redirect this phenomenon, because children can learn how to construct knowledge to reach it. It was concluded that it is important that children develop notions about the lives of the animals in the initial level, and this will collaborate to be sensitive towards nature.
\end{abstract}

Key words: Respect; animals; transversal; initial education; values 


\section{RESUMO}

As crianças, nos primeiros anos de vida, encontram-se no momento ideal para lançar as bases de seu sistema de crenças, alinhado aos objetivos sociais. 0 objetivo deste trabalho foi desenvolver a importância da noção de respeito aos animais como um tema transversal desde a educação inicial, para que um sistema de valores baseado na empatia seja promovido nas crianças. 0 esquema investigativo utilizado foi o documental com abordagem qualitativa e método hermenêutico. Com as informações coletadas, constatou-se que episódios de maustratos a animais ocorrem em todo o mundo, sendo a educação um instrumento que pode redirecionar esse fenômeno, pois as crianças em processo de aprendizagem estão prontas para construir conhecimentos. Concluiu-se que é importante que a criança na educação inicial tenha noções sobre a vida dos animais, e isso a ajudará a desenvolver sensibilidade para com a natureza.

Palavras-chave: Respeito; animais; transversalidade; educação inicial; valores

\section{INTRODUCCIÓN}

Durante el siglo XXI, hay un tema de discusión recurrente que aunque tratado en el siglo XX, no se encontró en el tapete tal como en la actualidad: el resguardo sobre los derechos de animales. Este es un punto álgido, porque de acuerdo con Quirola (2018), lamentablemente en ocasiones estos seres son víctimas de violencia, como la que parte por falta de empatía o compasión en algunos humanos. Esto ha sido estudiado porque se ha pretendido desentrañar la relevancia de la comunicación para sensibilizar a comunidades que ignoran el tema o lo asumen como algo trivial, por considerar que el ser humano tiene supremacía sobre otros entes. De hecho, este entramado se mueve en torno a temas delicados que incumben incluso a la educación porque:
El maltrato animal es un tema muy poco abordado, sin embargo, requiere mayor atención ya que estos actos enmascaran hechos violentos a terceros como: la violencia intrafamiliar, el hecho de agresión a otros sin precedente alguno es un reflejo de la disfuncionalidad social (Quirola, 2018, p. xviii).

En la construcción educativa, todo lo que se consolide al aprender colabora para generar cambios dentro de la sociedad, pues según Acosta et al (2020) "se concibe el aprendizaje como un proceso a través del cual se consiguen o modifican no solo conocimientos, sino también habilidades, conductas y valores" (p. 16). Por ello la educación juega un papel preponderante para que el individuo edifique su bloque ético conforme a lo que refiere al deber ser, pero también de acuerdo a su propio sistema de creencias, por lo que éste debe ser orientado para que no se pervierta. En el artículo 26.2 de la Declaración Universal de los Derechos Humanos de la Organización de Naciones Unidas, ONU (1948), se estatuye que la educación "tendrá por objeto el pleno desarrollo de la personalidad humana..." (p.6), y ese desenvolvimiento podría ser ideal si se estructura con base en el respeto al medio ambiente $\mathrm{y}$ todos los seres vivos porque sienten, tienen necesidades e incluso quien escribe estas líneas estima que llegan a amar.

Dentro del sistema educacional, la etapa inicial representa el punto de partida para orientar a los niños en miras a que en un futuro sean ciudadanos de bien, pues se asume como un "...proceso continuo y permanente de interacciones y relaciones sociales de calidad, oportunas y pertinentes 
que posibilitan a los niños y a las niñas potenciar sus capacidades y desarrollar competencias para la vida" (Ministerio de Educación Nacional de Colombia, 2014, p. 1). Así hay que desglosar la importancia del refuerzo de valores positivos en este nivel, que en este caso atendría al respeto a los animales como un tema transversal en el currículo, de manera que se estime su acción entre los infantes como replicadores sociales para un cambio en cuanto a la percepción de que toda vida debe ser protegida.

A tenor de lo expuesto, en el proceso investigativo ejecutado se consideraron las siguientes interrogantes: ¿en qué consiste el respeto a los animales?; ¿cómo incide la educación inicial en la construcción de un sistema de valores para las personas?; ¿qué aporte se podría generar para promover al respeto a los animales como un tema transversal en valores para la educación inicial? Y de acuerdo con dichas interrogantes, se construyeron los propósitos u objetivos de este documento.

En consonancia con lo indicado de forma antecedente, el propósito general de esta nota, es analizar el respeto a los animales como tema transversal en valores para la educación inicial. Igualmente, los propósitos específicos de la investigación son: 1) Expresar el alcance sobre la noción de respeto a los animales; 2) determinar el papel de la educación inicial en la formación en valores y, 3) explicar algunas consideraciones para la promoción del respeto hacia los animales como valor desarrollado en educación inicial.

\section{MÉTODO}

Esta investigación se elaboró con un enfoque cualitativo y siguiendo diseño documental. Según Sánchez el proceso de indagación que sigue esta perspectiva (2019), “...se sustenta en evidencias que se orientan más hacia la descripción profunda del fenómeno con la finalidad de comprenderlo..." (p. 104), y en este caso el acontecimiento es la implementación del respeto a los animales para consolidar valores en educación inicial. La revisión de documentos se realizó observando artículos científicos, libros, memorias científicas, normas jurídicas y reseñas periodísticas en medios prestigiosos. Para ello se utilizó la lectura en profundidad, el subrayado y el resumen como técnicas operacionales en el manejo de fuentes documentales. En cuanto a los criterios de selección para los textos manipulados en aras de hacer realidad a este trabajo, sistemáticamente se consideraron los siguientes:

a) Los documentos en primer lugar pasaron por un proceso de revisión para determinar cuáles podrían ser pertinentes para la investigación en cuanto a temática, escogiéndose en un primer proceso de descarte los que desde distintas perspectivas se vincularon con el problema escogido.

b) Como mínimo, el $50 \%$ de las fuentes informativas escogidas debían constar de documentos con un máximo de cinco (5) años de antigüedad con respecto al año de la elaboración del artículo (2020).

c) Los textos elegidos debían tener primordialmente carácter científico informativo formal, así que se procedió a decantarse mayormente por artículos contenidos dentro de revistas científicas e indexadas dentro de bases de datos e índices como SciELO, Scopus, Dialnet, Redalyc y similares como repositorios 
universitarios. Por otra parte, fueron útiles trabajos de grado y tesis doctorales encontradas en sitios web de casas de estudio superior americanas y europeas.

d) También sirvieron a los efectos de este trabajo y para obtener datos actualizados, artículos periodísticos digitales que debían encontrarse alojados y elaborados dentro de sitios pertenecientes a agencias noticiosas nacionales e internacionales, que aparte de su fama, debían contar con insignia de verificación en redes como Instagram, Facebook o Twitter, pues esta es una forma de confirmación de la seriedad que consideran las plataformas digitales para replicar las informaciones indicadas por estos entes.

e) Además los libros impresos utilizados debían estar apadrinados por editoriales de prestigio.

f) La información que no constaba de artículos científicos ni periodísticos, provino de websites oficiales de organismos públicos y entes también prestigiosos.

La fiabilidad de la información se determinó gracias a los criterios supra, pues las fuentes de donde se extrajeron los datos están caracterizadas por seriedad y aceptación entre las comunidades científica y no científica. Para analizar lo recabado, se implementó el análisis crítico con aplicación del método hermenéutico interpretativo de textos, sobre el cual indica Brito (2015) que “...el investigador analiza los distintos fenómenos de la realidad obtenidos $y$ registrados por otros investigadores en fuentes documentales" (p. 8).
Por otra parte, la información fue recuperada de la siguiente manera: 1 . Uso de internet como herramienta para acceder a las bases de datos consultadas, con utilización de software para la lectura de aquellos documentos encontrados bajo formato de PDF y 2) los textos impresos se extrajeron de la biblioteca personal de quien escribe este texto. De acuerdo con lo recabado, se desprendieron categorías que ayudaron a construir los propósitos y el esquema de este trabajo. Las que resultaron de la lectura y reflexión realizadas por el autor de acuerdo con aplicación de los procesos del pensamiento y aplicando destrezas investigativas desarrolladas a través de su experiencia en docencia, fueron:

1. Respeto a los animales: Acepción de los derechos animales como existentes y tangibles con su correcto ejercicio.

2. Valores: Eje de las percepciones éticas que tienen los seres humanos hacia otras vidas.

3. Educación inicial: Etapa dentro de la cual se desarrollaron las premisas planteadas.

4. Empatía: Desarrollo de capacidades para comprender las situaciones de otros.

Una vez identificadas las categorías investigativas, se determinó el boceto sobre los puntos que se habrían de abordar. Estos se adaptaron a los propósitos u objetivos de investigación, los cuales fueron ordenados de manera progresiva de acuerdo a los verbos de procesos cognitivos adecuados a la taxonomía de Bloom, conforme se tratara de pautas de dominio cognoscitivo en cuanto a: conocimiento, comprensión, aplicación, análisis y síntesis. 


\section{DESARROLLO Y DISCUSIÓN}

\section{Algunas consideraciones sobre el respeto hacia los animales}

En distintos países se reconocen cada año eventos lesivos de los derechos animales. En Perú, Morón (2019) considera que "se ha tocado mucho el tema del maltrato animal en estos últimos meses, debido a la incrementación de la misma, siendo realizado por algunos 'seres humanos', motivo por el cual...exigen justicia para tales actos" (pp. 6,7), mientras que en Ecuador, el ya citado Quirola (2018) en el momento de investigar el tema, indicaba que se conocían más de 500.000 casos que incluían abandono animal y se incrementaban con el transcurrir del tiempo. En Estados Unidos, conforme a Europa Press (2019), el FBI informó sobre 3226 casos de crueldad animal para 2017, aclarando que sus perpetradores eran: $84 \%$ adultos, $56 \%$ hombres, $65 \%$ personas blancas, al tiempo que sugería que se debía capacitar a los agentes de los cuerpos de seguridad para que dieran la misma importancia a los delitos contra animales y personas. Además, en Venezuela se han registrado episodios dantescos de tortura animal como el cometido por una fémina y reseñado por El Nacional (2020) por el cual “...será imputada por la comercialización de pornografía denominada Animal Crush Fetish. Este fetiche consiste en el aplastamiento y muerte de animales como forma de parafilia" (p.1).

Así se han señalado ejemplos ilustrativos de que el fenómeno del maltrato animal se suscita mundialmente, lo cual refuerza la noción de que debe ser combatido en aras de respetar a los animales a través de la protección de sus derechos, que consisten en “...principios y normas destinado a brindar una protección jurídica al animal de especie distinta a la del ser humano, promoviendo y procurando su bienestar..." (Chible, 2016, p. 375). El respeto a esos derechos no sólo se circunscribe a hacerlo porque se tiene comprensión hacia la vida ajena, sino también porque existen instrumentos que avalan esta acepción. La Declaración Universal de los Derechos del Animal de la ONU (1976), estipula en sus artículo 1 que estos entes tienen derecho a la existencia, e igualmente propugna su respeto en el artículo 2, mientras que en el 3, establece que no deben ser sometidos a tratos crueles y así, establece una serie de prerrogativas hasta su artículo 14, indicando que incluso sus restos mortales merecen una digna disposición.

Si bien la Declaración no tiene carácter obligatorio, es motivo de inspiración para instrumentos jurídicos que sí lo son. Ejemplo de ello son el Código Penal Peruano de 1991, que considera el trato cruel hacia los animales como un delito contra las buenas costumbres o como referencia Deustche Welle (2020), la legislación promulgada en Brasil durante septiembre 2020, por la cual se castiga con prisión a los maltratadores de perros y gatos. En Chile, se ha contemplado igualmente en el Código Penal la sanción de presidio para episodios de crueldad animal (Chible, 2016), y estos son solo algunos casos donde se evidencia que la garantía a la integridad animal debe ser moral y un deber normativo. Aparte como psicóloga, López (citada por El Tiempo, 2018) estima que el buen accionar hacia estos seres es una forma ideal de cultivar principios y sentimientos como la compasión, la bondad, responsabilidad y amor por la vida.

También debe recordarse que "en Estados Unidos un $68 \%$ de las familias conviven con un animal de compañía...En 
Europa se calcula que 75 millones de hogares tienen algún tipo de mascota" (Calvo, 2017, p.12), y así se observa que existe sinergia entre humanos-animales, siendo trascendental que se establezca un sistema de resguardo a esas vidas que conviven con familias. Adicionalmente el respeto debe orientarse hacia animales salvajes, silvestres, de ganadería o de compañía, pues pueden ejercer acción social.

Cajal et al (2018), defienden la tesis de que desglosar el maltrato animal obedece a principios que van más allá de los intereses sociales de la actualidad, dentro de los cuales se plantea el bienestar como norte de convivencia. Además aclaran que la práctica de la zoofilia es una forma de crueldad hacia estos entes, pues se utiliza a los animales con fines sexuales. Del mismo modo estiman que el maltrato animal incluye acciones tendentes a causar dolor y estrés a esos seres vivos, lo cual envuelve a la falta de diligencia en sus cuidados. Sin embargo, para ellos no entran en esta categoría los hechos como: la cacería normada y permitida jurídicamente, la cría animal para el uso de su pelaje, la ganadería de forma extensiva, realización de experimentos científicos y en algunas sociedades, la realización de prácticas para la diversión como peleas de gallos, corridas de toros, entre otras. Esto obedece a que se toleran cuando son socialmente aceptadas y por lo tanto, permitidas, pues hay que recordar que ninguna conducta constituye delito si no se encuentra establecida en una ley de tal manera.

Cabe indicar que sobre el respeto a los animales, en España el Gobierno de Aragón (2018) impulsó una iniciativa para incluirlo como asignatura dentro de algunos centros educativos que decidieran atender a la convocatoria denominada programa "Mundo
Animal", pero no por ello se constituyó como un eje obligatorio dentro de todas las instituciones. En su planificación, se estimó la aplicación de este proyecto en los niveles de educación primaria, bachillerato y la educación especial, con lo cual se persiguió catalizar la sensibilidad hacia los animales, crear conciencia acerca del cuidado animal y transformar a los educandos en agentes de cambio para la protección de las especies en sus espacios de convivencia. Dentro de lo previsto, se incluyeron clases de 90 (noventa) minutos de duración a nivel de primaria y 1 (una) hora en secundaria. Sin embargo, es destacable aclarar el hecho de que esta unidad sólo se implementaría en las instituciones que lo solicitaran y por otra parte, la iniciativa no incluyó a la educación inicial.

Igualmente resulta oportuno indicar que de acuerdo con Europa Press (2019) también en el Reino de España, agrupaciones políticas han insertado ante el Congreso una sugerencia no legal de incluir al respeto y la empatía hacia animales en el currículo escolar. En la propuesta se sostiene que diferentes investigaciones científicas prueban relación directa entre la violencia animal e interpersonal, por lo que estiman que el acto educativo puede desde esta perspectiva, mejorar la convivencia pacífica de los humanos.

El resguardo de los animales implica no perder el contacto con la naturaleza, pues como señala Leyton (2014), "detrás de esos ojos siempre hubo un "alguien" que me inspiraba respeto, por el hecho de ser otro, con su propia vida como individuo" (p. 10). Por esto debe entenderse la importancia de la educación inicial para propugnar el fin de auxiliar a estos sujetos. 


\section{La educación inicial y promoción de valores sobre respeto a los seres vivos}

Se asume como educación inicial a "el período de cuidado y educación de los niños en los primeros años de su vida, que se produce fuera del ámbito familiar" (Egido, 2000, p. 120), y así marca el desarrollo psíquico y social de sus participantes. Las funciones a las que se adapta son: asistencia a infantes por medio de los hogares para cuidado o guarderías y la escolarización en instituciones. Igualmente, en esta etapa se promueve el desarrollo de la socialización e impulso de valores.

Por ello, Tomasini (2008) parte del criterio de que en la educación preescolar se produce el proceso de interacción con similares y otros actores pedagógicos. Dicha relación en los centros de educación debería situar los contenidos conceptuales, procedimentales y actitudinales en la preparación sobre valores socialmente aceptados. Todo esto ocurre porque:

Los primeros años de vida en el ser humano constituyen una etapa con características propias cuyas problemáticas se analizan vinculadas al desarrollo ulterior del sujeto; este periodo es considerado como el momento en que se sientan las bases para el desarrollo físico y espiritual del hombre. Es durante la primera infancia cuando se asimilan conocimientos, habilidades $\mathrm{y}$ hábitos; además, se forman capacidades y cualidades volitivomorales (Gutiérrez y Ruiz, 2018, p. 34).

Consecuentemente, los ejes transversales educativos pueden resultar determinantes para proveer los conocimientos deseados a los infantes en preescolar. Al implementar temas de ese corte que complementen los aprendizajes académicos, se estarían potenciando competencias para uso futuro en la construcción social. González y Martínez (2019), sostienen que el desarrollo de competencias es un camino dinámico que necesita diversidad de estrategias sistemáticas, insertadas en las actividades regulares y afines con el currículo, por lo que en educación inicial deben diversificarse las formas de procurar los valores, especialmente en cuanto al respeto hacia los animales. Esto es porque un tema transversal:

...Hace referencia a contenidos de enseñanza y aprendizaje que por su relevancia formativa no pueden confinarse en el ámbito de una determinada disciplina $o$ área curricular, sino que deben ser objeto de tratamiento en todas o en una pluralidad de ellas. (p. 144).

Lucini (2000), sostiene que la educación en valores pretende consolidar en los individuos un marco humano, libre $\mathrm{y}$ solidario, por lo que en la transversalidad, los tópicos se orientan a necesidades y realidades que son relevantes para las personas, de manera que se edifique una sociedad armoniosa, siendo que sus dimensiones educacionales son para: paz, igualdad, vialidad, sexualidad y salud, el consumidor, y la que ocupa a este texto que es la referida al ambiente, pues dentro de su cuidado entran flora y fauna. Sin embargo, resulta curioso que en los libros como recursos pedagógicos, se traten aspectos como funcionamiento de los organismos, entre otros, que resultan “...temas afines al bienestar animal. Pero en ningún caso se menciona el bienestar animal ni se hace alusión específica a este término en 
los contenidos" (Fernández y Mazas, 2016, p. 311 ), lo cual es una nota a acotar sobre lo importante que es dejar claro dentro del eje ambiental, que debe específicamente aducirse al respeto a los animales y rechazar su maltrato, pues esto puede desembocar incluso en daños irreversibles para los ecosistemas. Esto también ayuda a fortalecer las relaciones con estos seres y con los semejantes al sustentar la empatía. Incluso al incorporar animales en educación, es esencial que los niños comprendan que:

...Son seres sintientes, con capacidad para sufrir debido a nuestras acciones, sujetos de una vida que puede verse afectada para bien o para mal por el efecto de éstas; son seres conscientes del mundo y de lo que les ocurre, y eso tiene importancia para ellos (cfr. Regan, 2016). Estas circunstancias los hace objetos de consideración moral, lo cual implica restricciones en el modo como los tratamos, así como ciertas obligaciones que tenemos hacia ellos. Sus intereses no tienen que ceder automáticamente a los intereses humanos (Ortiz, 2016, p. 166).

Lo anterior puede lograrse si los infantes participantes en el sistema de educación inicial se acoplan a proyectos adaptados a tal fin. Pérez (2017), indica que el interés de los niños sobre los seres vivos puede despertarse al fomentar su curiosidad, especialmente si se incorporan animales no humanos a las actividades de aula para que perciban con sus sentidos cómo son. Esta iniciativa resulta aún más viable si colaboran en ella los padres de los estudiantes dotando de los recursos que estén a su alcance. La autora manifiesta que la atención a dichos seres puede proveerse si se enseñan aspectos de corte animalista como: clases, respeto propiamente dicho, sonidos, texturas y trabajo cooperativo, y puede incluirse su trato. Sin embargo, de acuerdo con Villalta (2018), no son sólo los educandos quienes tienen que desarrollar la sensibilización con respecto a las necesidades y el amor hacia los animales, sino que los docentes también deben estar contestes en tal cuestión, puesto que si motivan lograrlo, promoverían destrezas comunicativas, afectivas, sociales, espirituales, morales y cognoscitivas.

Independientemente del nivel educativo, desde el punto de vista del constructivismo, Alfaro (2000) apunta que el proceso de enseñanza es "un apoyo pedagógico o una mediación cognitiva que debe estar ajustada a la calidad, cantidad y continuidad a las necesidades reales que va experimentando el educando..." (p. 179) y es de esta manea que fundado el tales concepciones asumidas desde la realidad, el acto educativo tiene que procurar alternativas y propuestas palpables que sean capaces de redimensionar al mundo desde una perspectiva humana y con sensibilidad hacia el ambiente y sus partes, pues dentro de él han de convivir las personas. $\mathrm{He}$ aquí la relevancia en la importante labor de enseñar todos estos valores en la educación preescolar.

\section{Discusión}

El desarrollo de las habilidades para comprender que los animales son entes que tienen sensaciones al igual que los seres humanos, forma parte de lo que Ortega y Rodríguez (2009), han denominado como educación para el respeto a los seres vivos, que "...se refiere a la formación de valores para proteger a los animales y disminuir la violencia social" (p. 6), lo cual resulta cierto de acuerdo con los datos hasta aquí 
presentados. La sensibilización de los niños hacia la situación de los animales, es crucial para prevenir la consolidación de comportamientos inadecuados en los adultos, pues lo que tempranamente quede impreso en sus fueros internos, acondicionaría sus subsiguientes etapas de desarrollo y en parte se evitaría incluso el cultivo de criminales como la del caso venezolano señalado previamente, quien a priori podría decirse que por un esquema de antivalores, participó en actos de crueldad animal a cambio de dinero, lo cual le acarreó como consecuencia que ahora sea procesada judicialmente.

Es importante el desarrollo de la empatía hacia los animales desde el nivel inicial, pues de este modo se sembraría la semilla del dicho no hagas a otros lo que no quieres que te hagan a ti: al sensibilizar a los niños sobre el amor a la naturaleza y su obra, del mismo modo se condiciona que tal empatía se manifieste a todo lo que los rodea, lo cual es componente de la educación en valores durante el siglo XXI. Para lograrlo, es importante levantar propuestas a este respecto y de hecho, en fase de diagnóstico sobre la problemática escogida y aquí desarrollada, se determinó que ella se genera alrededor de todo el mundo en diferentes grados por medio de la lectura de noticias en redes sociales como Instagram o Twitter. Además la idea para el desarrollo de este artículo se inició con una imagen observada por el autor en Facebook, y cuya leyenda rezaba: la educación de los niños debe incluir amar y respetar a los animales, lo cual se complementó con el recuerdo de diversos episodios a lo largo de la vida de quien escribe estas líneas, durante los cuales en ocasiones, presenció manifestaciones de crueldad animal por parte de otras personas hacia esos seres indefensos.
Aparte de lo indicado, se identificó al contexto dentro del cual podría implementarse como aporte el contenido aquí presentado y siendo que esta investigación no pretendió desentrañar un caso específico, sino aludir directrices generales porque esta incidencia ocurre en diversos territorios alrededor del planeta tal y como se referenció en las bases teóricas, se determinó que la colaboración podría ser de interés universal y no sólo regional. Sin embargo, las pautas genéricas concebidas deberían adaptarse por cada Estado de acuerdo a su realidad, pues distintos factores cambian de un lugar a otro, así como también esto dependerá de los planes curriculares, las políticas públicas y las leyes del país que se trate. Por ello, este trabajo propone que el Estado a través del currículo cimentado sobre ejes transversales, invite al docente a mediar el aprendizaje de los niños en torno a los siguientes componentes temáticos:

a) Componente 1: Clases de animales. Propósito: conocer la diversidad animal. Contenido: animales domésticos, de granja, de trabajo, fauna silvestre y salvaje. Ejemplos, diferencias en cuanto al trato y contacto con ellos.

b) Componente 2: Cuidado de los animales. Propósito: Describir los elementos básicos del cuidado animal. Contenido: cuidado en animales domésticos, de granja y de trabajo, alimentación, aseo y control sanitario. Cuidado del ecosistema de la fauna silvestre.

c) Componente 3. El respeto a los animales. Propósito: Comprender las razones por las cuales debe respetarse a los animales como seres vivos. Sensaciones en los animales, manifestaciones anímicas, reciprocidad en el buen trato hacia los 
animales, razones por las cuales no debe maltratarse a los animales, el amor hacia nuestras mascotas.

Estos componentes no son limitativos por lo que los docentes, en conjunto con la comunidad educativa e incluso los padres, podrían complementarlos para generar una experiencia más completa siempre que no vulnere el plan curricular a nivel macro. Entre las estrategias que podrían ejecutarse a estos fines se encuentran: uso de marionetas, canciones, construcción de esculturas con plastilina, papel $\mathrm{u}$ otro material, demostración, dramatizaciones, juegos, dibujos, pintar figuras, utilización de recursos audiovisuales como películas, visitas a centros animalistas e incluso se podrían implementar actividades como la americana show and tell (mostrar y contar), dentro de las cuales se muestren animales como las mascotas de los estudiantes o figuras de felpa para comentar las experiencias con ellas. Puede resultar muy importante la aplicación de la técnica de preguntas y respuestas para fomentar la empatía al plantearles a los niños: ¿cómo te sentirías si te tratan como a...?, ¿qué crees que siente este animal si...? Y similares, así como la gestión de proyectos. En cuanto a los recursos utilizables, grosso modo pueden ser:

- Financieros. Son partidas presupuestarias establecidas desde los centros para el aprendizaje, en miras a desarrollar el proceso. Además consta de aportes económicos procurados por distintos individuos a través de figuras como las donaciones y aparte incluyen a aquellos planificados dentro de los apoyos financieros estatales.

- Físicos. Se refieren a los objetos necesarios para realizar lo que se desea emprender.
En este caso incluye a libros, revistas, lápices, crayones, pintura, pinceles, papel, plastilina, etc., que igualmente pueden gestionarse con partidas presupuestarias de los centros educativos.

- Humanos. Son los sujetos que deseen o deban ejecutar las pautas antes apuntadas, tales como docentes, miembros de la comunidad educativa y de la comunidad general, educandos, funcionarios públicos y trabajadores privados que deseen participar, entre otros.

- Audiovisuales y tecnológicos. proyectores, computadores, equipos de sonido, televisores, celulares, equipos de DVD o blue ray, con los que incluso se puede trabajar con películas aleccionadoras o series como: La razón de estar contigo (a dog's purpose), Dumbo, Bambi, Peppa pig, y similares.

Al tratarse en este trabajo los temas transversales, se entiende que quien habrá de ejecutar el proceso educativo hacia los niños es principalmente el docente que convive día a día con ellos (incluso aunque en la actualidad lo haga en el marco de distanciamiento social por pandemia de COVID-19, que ha impulsado el uso de plataformas para aprendizaje a distancia, en caso que aplique). Esto es, porque se debe recordar que la transversalidad precisamente refiere al acoplamiento de lo que se desea facilitar, insertándolo de una manera u otra dentro de la mayoría de las demás actividades que se realicen en las aulas, aunque versen sobre temas distintos como habilidades matemáticas, artísticas, lingüísticas, entre otras. No quiere decir esto que eventualmente y cada cierto tiempo, no se solicite el apoyo de personal externo (como trabajadores de centros de protección animal, entre otros) para que 
soporten la enseñanza, lo cual incluso podría hacerse a través de la capacitación del personal docente con expertos en el tema. Esto resulta especialmente importante en los lugares donde se implementen los modelos por competencias, porque tal como en el caso de Venezuela, se ejecutan proyectos integradores dentro de las instituciones educacionales.

Debe destacarse además que si bien por su nivel de desarrollo cognitivo, se dificulta la enseñanza de los marcos jurídicos que protegen a los animales, por medio de la analogía puede lograrse algo similar: hay que reforzar en los niños que existen ciertas acciones que son inadecuadas dentro de sus hogares, y del mismo modo están otras que lo son con respecto a un animal, explicando con palabras sencillas las razones por las cuales equivalen a las normas de sus entornos. Un buen referente para lograr las metas planteadas es el sistema educativo japonés: De a acuerdo con Arana (2015) allí se propugna el civismo haciendo que los niños cuiden de su integridad y la de los demás, se interesen por ellos mismos y por el ambiente realizando labores colaborativas, de limpieza, incluso con cultivo de flores, otras plantas y la crianza de animales, especialmente en entornos rurales. Esto los enseña a valorarse a sí mismos pero desarrollan además la empatía con respecto a todo su mundo circundante. Es importante recalcar que el irrespeto a los animales puede conducir a comportamientos de carácter criminal, pues como se estima en Cajal et al. (2018):

El abuso y la crueldad hacia los animales se encuentran presentes en los trastornos que manifiestan conductas antisociales, como el trastorno de conducta infantil o el Trastorno Antisocial de la Personalidad (TAP) en la vida adulta (APA, 2002). A su vez, se configuran como conductas criminales y han sido asociadas a diferentes formas delictivas y de violencia interpersonal (p.113).

Asimilando lo anterior a una variante incluso clínica en la forma de vulneración a los derechos de los animales, e igualmente a la integridad e incluso la vida de los mismos, resulta consecuente alinearse con las concepciones de los autores supra, porque esta manifestación de violencia incluso podría escalar hasta dirigirse a otros seres humano, a pesar de que episodios de abuso animal pueden generarse en la evolución general de un niño. Sin embargo para quien ha redactado este artículo, por simple lógica la educación sobre respeto a un animal puede contraponerse a este fenómeno y por ello, constituir un paliativo o factor preventivo dentro de tales situaciones, cuando las razones de su ejecución no sean de carácter médico. Esta es una forma de potenciar el sistema de valores de los infantes, de acuerdo con los criterios expresados en esta investigación. Todo lo que pueda desarrollarse en los niños desde las más tempranas edades de la escolaridad, es un agregado para lograr una vida digna propia y de los miembros de sus entornos.

\section{CONCLUSIONES}

Conforme con los objetivos planteados en el texto, se concluyó que:

El respeto a los animales comporta la comprensión de sus cualidades como seres vivos que en muchas ocasiones ayudan a los humanos.

La educación inicial coadyuva a la consolidación de valores en los niños, pues es en esta etapa cuando se encuentran prestos a la construcción de aprendizajes que podrá aplicar en el futuro. Por ello la implementación 
de temas transversales como el respeto a los animales es fundamental en el marco de la práctica curricular.

Aplicando distintas estrategias puede lograrse que los infantes comprendan la importancia del respeto hacia los animales, lo cual es determinante para que sea empático y sensible ante el ambiente que los circunda.

En diversos territorios se establece la protección animal a través de sistemas jurídicos, por lo tanto la educación puede ser un importante aliado no sólo para dar a conocer estas pautas, sino también para educar a los entes responsables de implementarlas, de manera que se enriquezcan o en caso de no existir, que se elaboren. Si curricularmente se incorpora o desarrolla el respeto a los animales como uno de los temas transversales en valores desde la educación inicial, se podría potenciar esa cultura de resguardo y empatía hacia seres vivos no humanos lo cual podría decantarse en importantes cambios para las sociedades a futuro, impactando incluso en sus espectros normativos, pues hay que recordar que el Derecho se asume como toda norma jurídica que rige las conductas sociales humanas.

Igualmente en el marco de los aportes que se producen por procesos del pensamiento al elaborar investigaciones académicas, se presenta la siguiente reflexión final: Como amante de los animales, quien ha escrito estas líneas, pretende que esta colaboración sirva para plantear potenciales investigaciones sobre el rol de los niños en el aprendizaje sobre el respeto hacia los seres vivos, pues en cuanto a artículos científicos, este tópico parece insuficientemente explorado con respecto a otros temas. Quien haya podido apreciar una vida, o quien haya compartido con una mascota como un amigo dentro del hogar, podrá identificarse con este interés porque seguramente habrá experimentado alegrías y también tristezas que provocaron lágrimas que solo ella pudo limpiar. Los niños son quienes progresivamente se encargarán de elevar a la sociedad y si desde pequeños se les enseña a resguardar a los que se encuentran en indefensión de una forma u otra, se catalizará la conformación de ciudadanos de bien, comprensivos y colaboradores que hagan del planeta Tierra un mejor lugar para vivir.

\section{REFERENCIAS}

Acosta, P., Cadena, D., Hinojosa, F., López E., Ortiz, D., Ramos-Galarza, C., Rubio, D. (2020). Autogestión del aprendizaje del universitario: un aporte en su construcción teórica. Revista ESPACIOS, 41 (18),

p.

16. https://www.revistaespacios.com/a20v4 1n18/20411816.html\#

Alfaro, M. (2000). Evaluación del aprendizaje. Caracas: FEDUPEL.

Brito, Argenis (2015). Guía para la elaboración, corrección y asesoramiento de trabajos de investigación. San Tomé: Universidad Nacional Experimental Politécnica de la Fuerza Armada Bolivariana.

Cajal, M., Díaz, M., Irurzún, J., Nadal, Z., Reyes, P. Solimena, N. Widensky, B. (2018). Psicopatía criminalística y maltrato animal. En M. Díaz Videla \& M.A. Olarte (Eds.), Antrozoología, multidisciplinario campo de investigación (pp. 112-133). Buenos Aires: Editorial Akadia.

Calvo, P. (2017). El vínculo entre el ser humano $y$ los animales: aspectos psicológicos y psicopatológicos (tesis doctoral, Universitat Autónoma de Barcelona). Recuperado de https://tinyurl.com/y6bofnst

Chible, M. (2016). Introducción al Derecho Animal: Elementos y perspectivas en el desarrollo de una nueva área del Derecho. Ius et Praxis, 22(2), pp. 373-414. https://dx.doi.org/10.4067/S071800122016000200012 
Deustche Welle (19 septiembre, 2020). Bolsonaro aumenta castigos a maltratadores de animales (aunque no quería) [artículo web]. Recuperado de https://tinyurl.com/y5ppbumz

Egido, I. (2000). La educación inicial en el ámbito internacional: situación y perspectivas en Iberoamérica y en Europa. Revista Iberoamericana de educación (22), pp. 119-146. https://tinyurl.com/y68qdxf4

El Nacional (19 octubre, 2020). FAES detuvo a mujer que se desnudaba y torturaba animales hasta asesinarlos [artículo web]. Recuperado https://tinyurl.com/y478no42

El Tiempo (29 junio, 2018). Respetar a los animales para ser mejores seres humanos [artículo web]. Recuperado de https://tinyurl.com/y6476zxv

Europa Press (6 enero, 2019). PSOE, Unidos Podemos y Ciudadanos quieren que la empatía y el respeto por los animales se enseñe en los colegios [artículo web]. Recuperado https://tinyurl.com/yy4f729z

Europa Press (22 enero, 2019). FBI publica los datos de maltrato animal de 2017 [artículo web]. Recuperado de https://tinyurl.com/y3xl7gpx

Fernández, R. y Mazas, B. (2016). El concepto de bienestar animal en el currículo de Secundaria y en los libros de texto de ciencias. Revista Eureka Sobre Enseñanza $Y$ Divulgación De Las Ciencias, 13(2), pp. 301-314.

https://revistas.uca.es/index.php/eureka/ article/view/296

Gobierno de Aragón (2018). Boletín Oficial de Aragón 227. ORDEN ECD/1852/2018, de 9 de noviembre, por la que se convoca el programa "Mundo Animal" en centros docentes públicos y privados concertados no universitarios de la Comunidad Autónoma de Aragón durante el curso 2018-2019 [página web]. Recuperado de https://tinyurl.com/y4qb7a8m

González, N. y Martínez, P. (2019). El dominio de competencias transversales en
Educación Superior en diferentes contextos formativos. Educação $e$ Pesquisa, 45, e188436. https://doi.org/10.1590/s16784634201945188436

Gutiérrez, A. y Ruiz, M. (2018). Impacto de la educación inicial y preescolar en el neurodesarrollo infantil. IE Revista de investigación educativa de la REDIECH, 9(17), $\quad$ pp. 33-51. http://www.scielo.org.mx/scielo.php?scri pt=sci_arttext\&pid=S244885502018000200033\&lng=es\&tlng=es.

Leyton, F. (2014). Bioética frente a los derechos animales: tensión en las fronteras de la filosofía moral (tesis doctoral, Universitat de Barcelona). Recuperado de https://tinyurl.com/y6f8olzc

Lucini, F. (2000). Temas transversales $y$ educación en valores. Madrid: Grupo Anaya.

Ministerio de Educación Nacional de Colombia (2014). ¿Qué es la educación inicial? [Página web]. Recuperado de https://tinyurl.com/y25xdmuw

Morón, M. (2019). La controversia del delito de abandono y actos de crueldad contra los animales domésticos y silvestres tipificados en el art. 206-A del Código Penal con la Ley $N^{\circ} 30407$, respecto a su calificación como seres sensibles (tesis de grado, Universidad Andina del Cusco). Recuperado de https://tinyurl.com/y5m2gbpn

ONU (1948). Declaración Universal de los Derechos Humanos [página web]. Recuperado de https://www.un.org/es/universaldeclaration-human-rights/

ONU (1976). Declaración Universal de los Derechos del Animal [página web]. Recuperado de https://www.ubu.es/aulade-paz-y-desarrollo/derechosuniversales/declaracion-universal-de-losderechos-del-animal

Ortega, D. y Rodríguez, O. (2009). Educación para el respeto a los seres vivos. San José: Coordinación Educativa y Cultural Centroamericana. 
Ortiz, G. (2016). Víctimas de la educación. La ética y el uso de animales en la educación superior. Revista de la educación superior, 45(177), $\quad$ pp. 147170. https://doi.org/10.1016/j.resu.2016. 01.010

Pérez, V. (2017). Los seres vivos en educación infantil: conocimientos y experiencias. . Tabanque. Revista Pedagógica, (30), pp. 95-114.

https://doi.org/10.24197/trp.30.2017.95 $-114$

Quirola, T. (2018). El uso de la fotografía monocromática en la concientización del maltrato animal en la ciudad de Ambato (trabajo de grado, Universidad Técnica de Ambato). Recuperado de https://repositorio.uta.edu.ec/bitstream/ 123456789/28965/1/Quirola\%20Tom\% C3\%A1s.pdf

Sánchez F. (2019). Fundamentos epistémicos de la investigación cualitativa y cuantitativa: Consensos y disensos. Revista Digital Investigación y Docencia 13 (1), pp. 101-122. http://www.scielo.org.pe/scielo.php?pid= S222325162019000100008\&script=sci_arttext Tomasini, M. (2008). La escolaridad inicial como contexto socializador: complejidad y conflictividad en la trama interactiva cotidiana. Revista Mexicana de Investigación Educativa, 13 (36), pp. 7-34. https://tinyurl.com/y2f22aho

Villalta, D. (2018). El cuidado de los animales domésticos en el desarrollo de la sensibilización en niños de 4 a 5 años de la Unidad Educativa Atenas (trabajo de grado, Universidad Técnica de Ambato). Recuperado de https://tinyurl.com/y2w99qk6 